\title{
Topical lidocaine inhibits spasm during colonoscopy: a double-blind, randomized controlled trial (with video)
}

\section{다 (1) $\odot$}

\author{
Authors \\ Lefor $^{3}$, Kazutomo Togashi ${ }^{1}$ \\ Institutions \\ 1 Aizu Medical Center Fukushima Medical University - \\ Coloproctology, Aizuwakamatsu, Fukushima, Japan \\ 2 Jichi Medical University - Pharmacology, Shimotsuke, \\ Tochigi, Japan \\ 3 Jichi Medical University - Surgery, Shimotsuke, Tochigi, \\ Japan
}

Daiki Nemoto', Kenichi Utano', Noriyuki Isohata', Shungo Endo', Kensuke Kumamoto', Taka-aki Koshimizu², Alan

submitted 17.8.2016

accepted after revision 6.2 .2017

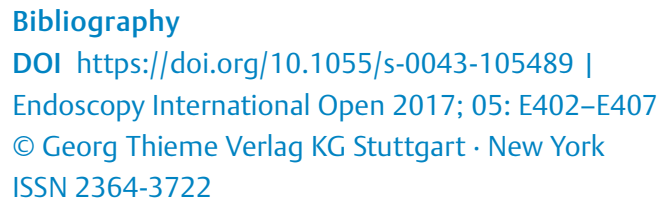

Corresponding author

Kazutomo Togashi, Aizu Medical Center, Fukushima Medical University - Coloproctology, 21-2 Maeda Tanisawa

Kawahigashi, Aizuwakamatsu, Fukushima

969-3492, Japan

Fax: 81-242752568

togashik@fmu.ac.jp

\section{ABSTRACT}

Background and study aims Topical peppermint oil prevents intestinal spasm, but can cause rebound spasm. Lidocaine hydrochloride, a local anesthetic, may work as an antispasmodic by blocking $\mathrm{Na}+$ channels. The aim of this study was to investigate the effect of topical lidocaine on the inhibition of colonic spasm during colonoscopy, compared with peppermint oil.

Patients and methods A randomized, controlled doubleblind trial was conducted in an academic endoscopy unit. Patients requiring endoscopic resection were randomly allocated to colonoscopy with topical administration of lidocaine $(n=30)$ or peppermint oil $(n=30)$. Similar vials containing different solutions were randomly numbered. Allocation was made based on the vial number. The solution used and the vial number were not revealed during the study. Two endoscopists performed all procedures using midazolam, without anticholinergic agents. When a pre-selected lesion was identified, the solution in the assigned vial was dispersed and the bowel observed for 5 minutes. The primary endpoint was the duration of spasm inhibition, and a secondary endpoint was the occurrence of rebound spasm stronger than before dispersion.

Results There were no significant differences in patient demographics. Spasm was inhibited in almost all patients in both groups, with a similar median duration (lidocaine $227 \mathrm{sec}$ vs. peppermint $212.5 \mathrm{sec}, P=0.508)$. In contrast, rebound spasm occurred less frequently in the lidocaine group (lidocaine $7 \%$ vs. peppermint $47 \%, P=0.001$ ). There were no adverse events or symptoms associated with administration of the solutions.

Conclusions The inhibitory effect of lidocaine is not superior to peppermint oil. However, lidocaine significantly decreases the frequency of rebound spasms.

\section{Introduction}

Hyoscine butylbromide and glucagon are used as antispasmodic agents during colonoscopy, since these agents relax the muscle tone in the gastrointestinal tract and aid in the conduct of colonoscopy [1-5]. However, their routine use is somewhat controversial due to various adverse effects (AEs) including miosis, palpitations, dry mouth, urinary retention (for hyoscine butylbromide), and hyperglycemia and reactive hypoglycemia (for glucagon) [6,7]. Peppermint oil [8-10] and warm water [11 -13] are considered alternatives to these agents for topical administration. Warm water infusion decreased patients' discomfort during colonoscopy but failed to suppress intestinal spasm [11 - 13]. In contrast, the inhibitory effect of topical peppermint oil has been validated by monitoring lumen volume [8]. The active component in peppermint oil is I-menthol, which blocks calcium channels in smooth muscle $[14,15]$. Peppermint oil solution dispersed through the colonoscopic channel prevents intestinal spasm, and drawbacks have not yet been reported. In our experience, however, the duration of effect of peppermint oil is rather short. Once the effect disappears, most patients report bothersome spasms. Repeated dispersion 
of peppermint oil does not control rebound spasms, which may necessitate termination of the procedure. This method has not been universally applied, and colonoscopists still seek an ideal antispasmodic agent with long-term duration but no AEs.

Lidocaine hydrochloride is generally used as a local anesthetic or antiarrhythmic agent and acts through blocking $\mathrm{Na}^{+}$channels, which are membrane-spanning proteins that form the basis of excitability in neuronal tissue, voluntary muscle and involuntary muscles including cardiac muscle $[16,17]$. In the gastrointestinal tract, this agent is speculated to work as an antispasmodic by relaxing smooth muscle tone. Topical use of lidocaine hydrochloride is contraindicated only for hypersensitivity to components of the formulation. Systemic administration of the agent, however, can cause serious side effects, including disorders of the central nerve system, making the agent quite different from hyoscine butylbromide or glucagon. Lidocaine hydrochloride may be an ideal antispasmodic agent during colonoscopic procedures. To our knowledge, however, there are no reports that lidocaine hydrochloride is effective as an antispasmodic agent during various endoscopic procedures. For ease of use, topical lidocaine hydrochloride would be best because submucosal injection is time-consuming and requires injection devices. If topical use is effective for preventing intestinal spasm, it may be useful in various situations, such as difficult intubation due to severe peristalsis, endoscopic resection with intractable peristalsis, and endoscopic submucosal dissection.

Peppermint oil is the only agent reported to effectively suppress intestinal spasm with topical use. A comparative trial of lidocaine hydrochloride and peppermint oil may provide useful data. Therefore, we undertook this study to investigate the inhibitory effects of topical lidocaine hydrochloride on colonic spasm during colonoscopy in comparison with peppermint oil.

\section{Patients and methods}

\section{Pilot study}

Before beginning this study, we performed a pilot study to identify the most effective concentration of lidocaine hydrochloride solution for topical use. In Japan, 5 different concentrations $(0.5 \%, 1.0 \%, 2.0 \%, 4.0 \%$ and $8.0 \%)$ of lidocaine hydrochloride solution are commercially available. In the pilot study, $1 \%$ lidocaine hydrochloride solution was not effective in preventing intestinal spasm but $2 \%$ was effective. Therefore, we used $2 \%$ lidocaine hydrochloride solution in the current study. The volume of solution dispersed was set at $30 \mathrm{~mL}$, following a previous report [8]. For sample size calculation, we measured the duration of the effect of $2 \%$ lidocaine hydrochloride solution and peppermint oil solution as a control.

\section{Study design/setting}

We conducted a prospective, double blind, randomized controlled trial to evaluate the effect of dispersed topical lidocaine hydrochloride on colonic spasm during colonoscopy at a tertiary-care hospital (Aizu Medical Center Hospital), compared with peppermint oil solution. The study was approved by the Institutional Review Board of Fukushima Medical University (No.
1805) and registered with the University Hospital Medical Information Network (UMIN000012352). Enrollment was from November 2013 to November 2014. The CONSORT (Consolidated Standards of Reporting Trials) guidelines were followed in reporting this study.

\section{Participants}

Both outpatients and inpatients aged 35 years or older who required endoscopy prior to resection and who were able to give informed consent were eligible for enrollment. Exclusion criteria included inflammatory bowel disease, allergy to lidocaine hydrochloride and inadequate (fair or poor) bowel preparation. The quality of bowel preparation was assessed according to the extent of mucosal visualization after suction of the fluid residue, following the Aronchick Bowel Preparation Scale: excellent ( $\geq 95 \%$ mucosal visualization); good ( $90-95 \%$ mucosal visualization); fair ( $80-90 \%$ mucosal visualization) and poor $(<80 \%$ mucosal visualization) [18]. None of the patients enrolled had participated in a previously published study. The authors enrolled all participants.

\section{Intervention and randomization}

Eligible patients were randomly assigned to one of two treatment groups: $30 \mathrm{~mL}$ of topically dispersed $2 \%$ lidocaine hydrochloride solution (LID group) or $30 \mathrm{~mL}$ of peppermint oil solution containing $240 \mathrm{mg}$ of I-menthol (PEP group). A random allocation sequence was generated using a random drawing of the vial number by a third party. To ensure concealed allocation, investigators were informed of the assignment only after identification of an eligible patient. These procedures ensured that investigators were blinded to the allocation sequence. Randomization was blinded such that neither the patient nor the investigator knew which medication was being administered. To maintain blinding, the study medication was labeled with a number pre-printed on the vial by a pharmacist.

\section{Procedure}

After undergoing a standard bowel preparation using polyethylene glycol plus ascorbic acid solution (Moviprep), colonoscopy was performed by one of the authors (ND or UK) using midazolam and without administration of anticholinergic agents. The endoscopist wore a peppermint-scented mask to limit olfactory sensation. To correctly mark the time when the solution was dispersed on the recorded digital video, $0.5 \mathrm{~mL}$ of $0.2 \%$ indigo carmine solution was added. The lesion of interest was selected in advance. If multiple lesions were identified during a previous colonoscopy, the most significant lesion was selected for the study. When the selected lesion was identified, $30 \mathrm{~mL}$ of the assigned solution was applied through the side-channel of the instrument using a $30-\mathrm{mL}$ syringe. To spray all of the solution, $30 \mathrm{~mL}$ of air was immediately injected into the channel using the same syringe. The assigned solution was dispersed as an aerosol and the area observed for exactly 5 minutes. Occurrence of intestinal spasm was defined as spasm of one-third or greater of the circumference of the lumen. The operator rated the inhibitory effect on intestinal spasm in real time and recorded the ratings after each procedure. When intestinal spasm was 
suppressed in less than one-third of the circumference of the lumen, "inhibition of spasm" was defined as present. The operator also rated occurrence of "rebound spasm." When intestinal spasm stronger than before spraying the solution occurred within 5 minutes of the observation time, "rebound spasm" was defined as present. The procedure was recorded on digital video. To identify occurrence of $A E$, we carefully monitored vital signs, oxygen saturation and cardiac rhythm during the colonoscopic procedure and nursing staff followed patients until they completely awakened from sedation.

\section{Outcome measures}

After completion of all 60 colonoscopy procedures, 4 authors (ES, KK, IN, and TK) measured the latency to spasm inhibition and duration of spasm inhibition, still blinded to the study medication used. Latency to spasm inhibition was the time between dispersion of the solution and start of the inhibition. The primary outcome measure was "duration of spasm inhibition" meaning the time spent in inhibiting colonic spasm within 5 minutes of the observation time. Secondary outcome measures were inhibition of spasm, rebound spasm, adverse events, and symptom associated with dispersion of the solution, all were rated in real time and recorded after each procedure. Also, latency to spasm inhibition rated by evaluation of the digital video was used as secondary outcome.

\section{Sample size calculation}

Sample size was calculated based on data from the pilot study. It was assumed that the duration of spasm inhibition is $240 \mathrm{sec}-$ onds with a standard deviation of 90 seconds. To detect a difference of at least 80 seconds between groups (33\%) using the Student's $t$ test with a 2 -sided alpha error of 0.05 and power of $0.80,21$ patients in each group of the study were required. Because the duration of effect for a small number is non-parametric data, comparison is made using the Mann-Whitney $U$ test. To account for drop-outs, the sample size in each arm was set at 30 patients in this study.

\section{Statistical methods}

Statistical tests compared measured responses for the 2 study groups (LID and PEP). For nominal data, comparisons were made using Fisher's exact test or the chi-square test for equality of proportions. When the data were ordinal or decidedly non-normal, the Mann Whitney $U$ test for trends was used to compare the distribution of responses. All $P$ values were twotailed. $P$ values less than 0.05 were considered to indicate statistical significance. All statistical analyses were performed with Intercooled Stata $13.0^{\circledR}$ for Windows (Stata Corp., TX, United States).

\section{Results}

Study participants included 60 patients ( $\mathbf{F i g} \mathbf{1}$ ). There were no significant differences in age, gender, hospitalization, body mass index, dosage of midazolam, bowel cleansing level or method of endoscopic resection comparing the LID $(n=30)$ and PEP groups $(n=30)$ ( $\triangleright$ Table 1$)$. No participants were ex-
Enrollment $(n=60)$

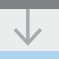

\section{Double blinded randomization \\ - Preparation in pharmacy \\ Similar vials containing different solutions \\ Random numbering}

\section{- Allocation}

A random sequence was generated using a random drawing of the vial number by a third party.

- Colonoscopy

Two colonoscopists

Peppermint flavored mask to prevent recognition of agent used

No administration of anticholinergic agents but use of midazolam

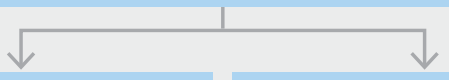

Lidocaine group $(n=30)$

Peppermint group $(\mathrm{n}=30)$

$\checkmark$

\section{Outcome measurements}

- Primary endpoint: duration of spasm inhibition

- Secondary endpoints: latency to spasm inhibition, inhibition of spasm, rebound spasm, adverse events and symptoms associated with dispersion of the solution.

Fig. 1 Study flow diagram.

cluded due to inadequate (fair or poor) bowel preparation. The distribution of anatomical areas of the colon examined was similar in the 2 groups.

In regard to analyzing latency to spasm inhibition and duration of spasm inhibition, 2 patients were excluded due to recording trouble and the data for 58 patients were analyzed. The latency to spasm inhibition and duration of spasm inhibition are shown in > Table 2 . In the LID group, the latency to spasm inhibition tended to be shorter and the duration of spasm inhibition longer than in the PEP group. The inhibitory effect continued at the end of the observation time in 16 patients in the LID group and 13 patients in the PEP group. There were no statistically significant differences. There were no AEs or symptoms associated with dispersion of the solution during the study.

The inhibitory effect on colonic spasm during colonoscopy is shown in $>$ Table 3 . In both groups, the inhibitory effect occurred in almost all patients, and only 1 patient in each group did not show any effect. In contrast, rebound spasm was significantly less frequently observed in the LID group (LID: 2/30, 7\%, PEP: $14 / 30,47 \%$; $P=0.001)$.

An example of a patient in the LID group is shown in $>$ Video 1. After dispersion of lidocaine solution with blue staining, intestinal spasm disappeared immediately and rebound spasm did not occur. 
- Table 1 Patient demographic data.

\begin{tabular}{|c|c|c|c|c|}
\hline & & $\begin{array}{l}\text { Peppermint oil } \\
n=30\end{array}$ & $\begin{array}{l}\text { Lidocaine hydrochloride } \\
n=30\end{array}$ & $P$ value \\
\hline Age, years & Median (range) & $73(50-95)$ & $71(39-85)$ & $0.32^{1}$ \\
\hline \multirow[t]{2}{*}{ Gender } & Female, n (\%) & $12(40)$ & $12(40)$ & $1.00^{2}$ \\
\hline & Male, n (\%) & $18(60)$ & $18(60)$ & \\
\hline \multirow[t]{2}{*}{ Hospitalization } & Yes, n (\%) & $20(67)$ & $24(80)$ & $0.24^{2}$ \\
\hline & No, n (\%) & $10(33)$ & $6(20)$ & \\
\hline Body mass index, $\mathrm{kg} / \mathrm{m}^{2}$ & Median (range) & $23.1(18.6-29.7)$ & $24.3(18.2-35.3)$ & $0.12^{1}$ \\
\hline Dose of midazolam, mg & Median (range) & $4(1.5-10)$ & $4(2-12)$ & $0.28^{1}$ \\
\hline \multirow[t]{2}{*}{ Bowel cleansing level } & Excellent, n (\%) & $18(60)$ & $16(53)$ & $0.80^{2}$ \\
\hline & Good, n (\%) & $12(40)$ & $14(47)$ & \\
\hline \multirow[t]{4}{*}{ Method of endoscopic resection } & ESD, n (\%) & $12(40)$ & $15(50)$ & $0.51^{3}$ \\
\hline & Conventional snare polypectomy, n (\%) & $7(23)$ & $6(20)$ & \\
\hline & EMR, n (\%) & $4(13)$ & $6(20)$ & \\
\hline & none $e^{4}, \mathrm{n}(\%)$ & $7(23)$ & $3(10)$ & \\
\hline $\begin{array}{l}\text { ESD, endoscopic submucosal dissect } \\
{ }^{1} \text { Mann-Whitney U test } \\
{ }^{2} \text { Fischer's exact test } \\
{ }^{3} \text { Chi-square test }\end{array}$ & EMR, endoscopic mucosal resection. & & & \\
\hline
\end{tabular}

- Table 2 Latency to spasm inhibition and duration of spasm inhibition.

\begin{tabular}{|c|c|c|c|c|}
\hline & & Peppermint oil & Lidocaine hydrochloride & $P$ value $^{1}$ \\
\hline \multirow[t]{2}{*}{ Latency to spasm inhibition, sec } & Median & 48.5 & 43.5 & 0.445 \\
\hline & 25th -75 th percentiles & $28-65.8$ & $23.8-58.5$ & \\
\hline \multirow[t]{2}{*}{ Duration of spasm inhibition, sec } & Median & 212.5 & 227 & 0.508 \\
\hline & 25th-75th percentiles & $105.5-249$ & $113-256.3$ & \\
\hline
\end{tabular}

- Table 3 Inhibitory effect on colonic spasm during colonoscopy.

\begin{tabular}{|c|c|c|c|c|}
\hline & & Peppermint oil & Lidocaine hydrochloride & $P$ value $^{1}$ \\
\hline \multirow[t]{2}{*}{ Inhibition of spasm } & None & 1 & 1 & 1.00 \\
\hline & Present & 29 & 29 & \\
\hline \multirow[t]{2}{*}{ Rebound spasm } & None & 16 & 28 & 0.001 \\
\hline & Present & 14 & 2 & \\
\hline
\end{tabular}




\section{Discussion}

This is the first report demonstrating that topical administration of lidocaine hydrochloride solution inhibits intestinal spasm. Although 1 patient in each group did not have inhibition of intestinal spasm, topically dispersed lidocaine and peppermint both inhibited intestinal spasm in almost all patients. There was no significant difference in the duration of spasm inhibition comparing the 2 groups. This suggests that the inhibitory effect of lidocaine hydrochloride compares favorably with peppermint oil, well known as an antispasmodic agent for topical use. This study also demonstrates that rebound spasm in patients treated with topical lidocaine occurs less frequently during the 5-minute observation period, compared with patients treated with peppermint. This may be useful for colon spasms seen during intubation/extubation, observation for significant lesions, and advanced therapeutic procedures.

To reduce measurement bias, the time to observing the effect as well as duration was assessed on digital video after completion of all procedures, with clear definitions used, as described in Patients and Methods. There were no statistically significant differences observed in the time to initiating the effect or duration of effect. This may be partially attributed to a short observation time. The peak effect of lidocaine was observed at 2 to 5 minutes after dispersion in the pilot study, so the 5-minute observation in this study may have been too short to reach statistical significance for duration of effect. Indeed, even at the end of the observation period, the effect was noted to continue in most patients.

Lidocaine hydrochloride is an amide local anesthetic known to have a longer effective period for inhibiting sodium channels than ester-type local anesthetics such as procaine. The anesthetic effect of lidocaine $(2 \%-10 \%)$ reportedly lasts for 30 to 45 minutes in mucous membranes [19]. The long-lasting and extensive anesthesia produced by lidocaine is related to the decreased incidence of rebound spasm. In contrast to peppermint, therefore, topical administration of lidocaine is more suitable for advanced therapeutic colonoscopy procedures including colonic endoscopic submucosal dissection. We have preferably used topical lidocaine during colonic endoscopic submucosal dissection and no significant complications have been experienced to date (data not shown). The depth of infiltration and sites of action are different for peppermint oil and lidocaine hydrochloride. As shown in - Fig. 2a, I-menthol, which is the main active component of peppermint oil, infiltrates up to the muscularis propria layer and blocks the voltage-dependent calcium channels of the smooth muscle and also those channels of nerves in the submucosal layer and the muscularis propria layer [20]. In contrast, lidocaine blocks sodium channels of the nerves only in the mucosal layer ( $\$$ Fig. $\mathbf{2 b}$ ) because it cannot infiltrate into the submucosal layer or deeper $[17,19]$. The sensory nerves of the mucosal layer originating from visceral nerves are regarded as minor contributors to intestinal peristalsis. The current study suggests that sensory nerves of the mucosal layer greatly contribute to this system [21]. It is reasonable that topical administration of lidocaine inhibits intestinal spasm induced mostly by colonoscopic movement, through

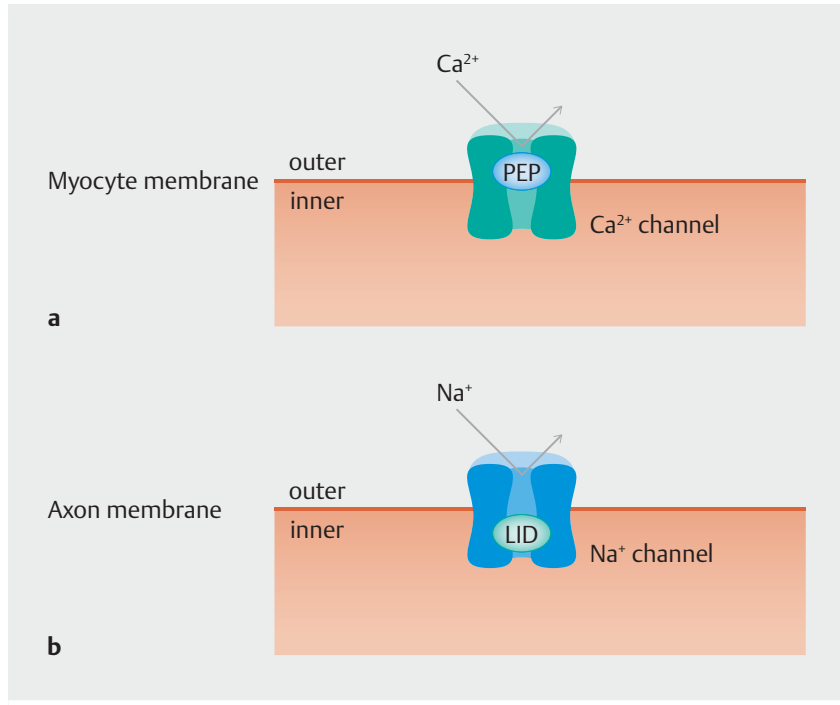

- Fig. 2 a Pharmacological mechanism of peppermint oil (PEP). b Pharmacological mechanism of lidocaine hydrochloride (LID).

blocking a feedback mechanism mediated by sensory nerves in the mucosal layer. In addition, lidocaine absorbed into the blood might act on the intestinal nerve plexus, but the effect would be negligible because the amount of lidocaine absorbed is minimal.

Some limitations of this study are acknowledged. Because this is the first report showing that topical lidocaine hydrochloride solution inhibits intestinal spasm, it should focus on the drug's inhibitory effect and not its superiority to peppermint oil. If one of the solutions used did not prevent intestinal peristalsis at all (for example, the dispersion of saline in a control group) the inhibitory effect of lidocaine would be clearly demonstrated in the current study. In addition to setting a control group, extension of the observation time to 10 minutes may have shown significant superiority in the duration of the effect comparing lidocaine to peppermint oil. Second, because only 2 endoscopists in a single center participated in the trial, generalizability of the results may be limited. Third, we did not

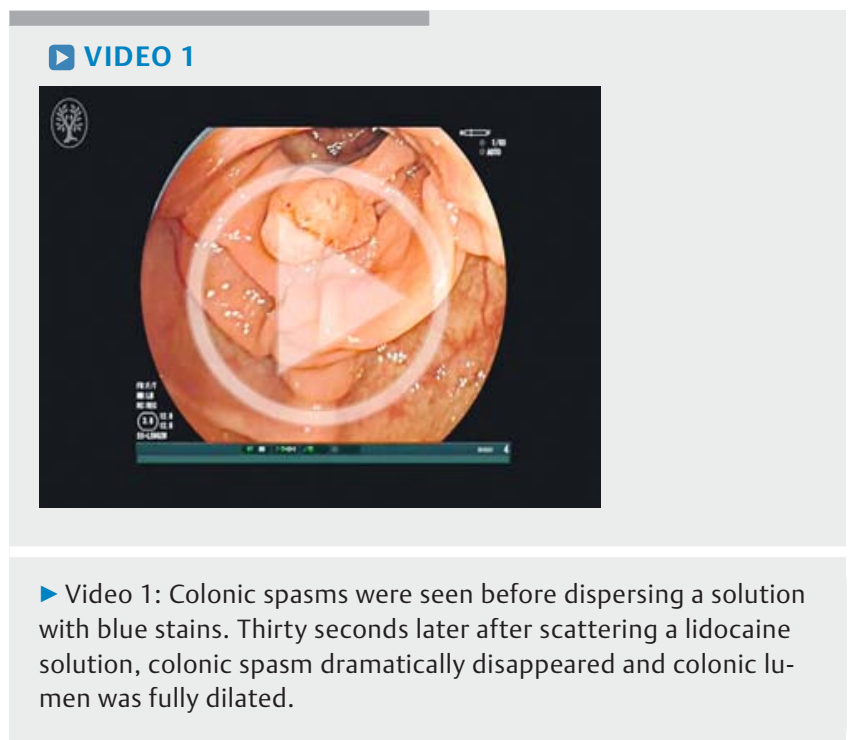


investigate the serum levels of lidocaine hydrochloride. Therefore, the pharmacokinetics of lidocaine has not been clarified based on topical use. These limitations should be addressed in a future study.

\section{Conclusion}

This study was a superiority trial, not a non-inferiority trial. A potential conclusion to draw would be that the inhibitory effect of lidocaine was not superior to peppermint oil; however, lidocaine significantly decreased the frequency of rebound spasms. Additional study of lidocaine as an antispasmodic agent is warranted.

\section{Acknowledgements}

We would like to thank Shinichi Sekimoto (Pharmacy, Aizu Medical Center Fukushima Medical University) for technical support, Toyomi Kamesaki, MD, PhD (Center for Community Medicine, Jichi Medical University, Japan) for his advice on this work, Professor Yoshikazu Nakamura (Department of Public Health, Jichi Medical University, Japan) for his advice on statistical analyses, and Ms. Jinko Kobayashi for administrative support.

\section{Competing interests}

None

\section{References}

[1] East JE, Saunders BP, Burling D et al. Mechanisms of hyoscine butylbromide to improve adenoma detection: A case-control study of surface visualization at simulated colonoscope withdrawal. Endosc Int Open 2015; 3: E636-641

[2] Ashraf I, Ashraf S, Siddique $S$ et al. Hyoscine for polyp detection during colonoscopy: A meta-analysis and systematic review. World ] Gastrointest Endosc 2014; 6: 549-554

[3] Marshall JB, Patel M, Mahajan RJ et al. Benefit of intravenous antispasmodic (hyoscyamine sulfate) as premedication for colonoscopy. Gastrointest Endosc 1999; 49: 720 - 726

[4] Mui LM, Ng EK, Chan KC et al. Randomized, double-blinded, placebocontrolled trial of intravenously administered hyoscine N-butyl bromide in patients undergoing colonoscopy with patient-controlled sedation. Gastrointest Endosc 2004; 59: $22-27$

[5] Norfleet RG. Premedication for colonoscopy: randomized, doubleblind study of glucagon versus placebo. Gastrointest Endosc 1978; 24: $164-165$
[6] Umegaki E, Abe S, Tokioka S et al. Risk management for gastrointestinal endoscopy in elderly patients: questionnaire for patients undergoing gastrointestinal endoscopy. J Clin Biochem Nutr 2010; 46: 73 80

[7] Yoshikawa I, Yamasaki M, Taguchi M et al. Comparison of glucagon and scopolamine butylbromide as premedication for colonoscopy in unsedated patients. Dis Colon Rectum 2006; 49: 1393 - 1398

[8] Asao T, Mochiki E, Suzuki $\mathrm{H}$ et al. An easy method for the intraluminal administration of peppermint oil before colonoscopy and its effectiveness in reducing colonic spasm. Gastrointest Endosc 2001; 53: $172-177$

[9] Hiki N, Kurosaka H, Tatsutomi Y et al. Peppermint oil reduces gastric spasm during upper endoscopy: a randomized, double-blind, doubledummy controlled trial. Gastrointest Endosc 2003; 57: 475-482

[10] Grigoleit HG, Grigoleit P. Gastrointestinal clinical pharmacology of peppermint oil. Phytomedicine 2005; 12: 607-611

[11] Church JM. Warm water irrigation for dealing with spasm during colonoscopy: simple, inexpensive, and effective. Gastrointest Endosc 2002; 56: 672-674

[12] Lee $\mathrm{H}, \mathrm{Kim}$ JJ, Min BH et al. Effectiveness of warm water consumption to reduce patient discomfort during colonoscopy: a randomized controlled trial. Am J Gastroenterol 2009; 104: 2935 - 2941

[13] Lee BY, Katon R, Herzig D et al. Warm water infusion during sedated colonoscopy does not decrease amount of sedation medication used. Gastrointest Endosc 2012; 76: $1182-1187$

[14] Hawthorn M, Ferrante J, Luchowski E et al. The actions of peppermint oil and menthol on calcium channel dependent processes in intestinal, neuronal and cardiac preparations. Aliment Pharmacol Ther 1988; 2: $101-118$

[15] Hills JM, Aaronson PI. The mechanism of action of peppermint oil on gastrointestinal smooth muscle. An analysis using patch clamp electrophysiology and isolated tissue pharmacology in rabbit and guinea pig. Gastroenterology 1991; 101: 55-65

[16] Sheets MF, Fozzard HA, Lipkind GM et al. Sodium channel molecular conformations and antiarrhythmic drug affinity. Trends Cardiovasc Med 2010; 20: 16-21

[17] Fozzard HA, Sheets MF, Hanck DA. The sodium channel as a target for local anesthetic drugs. Front Pharmacol 2011; 2: 68

[18] Aronchick CA, Lipshutz WH, Wright SH et al. Validation of an instrument to assess colon cleansing [abstract]. Am J Gastroenterol 1999; 94: 2667

[19] Catterall WA, Mackie K. Local Anesthetics. In: Brunton L, Lazo JS, Parker KL, editors. Goodman \& Gilman's The Pharmocological Basis of Therapeutics. 11th ed. New York: MacGraw-Hill; 2006: 369-386

[20] Amato A, Liotta R, Mule F. Effects of menthol on circular smooth muscle of human colon: analysis of the mechanism of action. Eur J Pharmacol 2014; 740: 295-301

[21] Dinning PG, Wiklendt L, Omari T et al. Neural mechanisms of peristalsis in the isolated rabbit distal colon: a neuromechanical loop hypothesis. Front Neurosci 2014; 8: 75 